Supporting Information (15 pages, 8 Figures, 3 Tables) for

\title{
Facile Prepolymer Formation with Ozone-Pretreated Grass Lignin by in situ Grafting of Endogenous Aromatics
}

\author{
Julian R. Silverman ${ }^{1}$, Andrew M. Danby ${ }^{1}$, Bala Subramaniam ${ }^{1,2^{*}}$
}

${ }^{1}$ Center for Environmentally Beneficial Catalysis, University of Kansas, 1501 Wakarusa Drive, Lawrence, Kansas 66047, United States

${ }^{2}$ Department of Chemical and Petroleum Engineering, University of Kansas, $1530 \mathrm{~W}$. 15th Street, Lawrence, Kansas 66045, United States

${ }^{*}$ Corresponding Author: bsubramaniam@ku.edu

Content

Analytical Methods for NMR, GPC, GC-FID, TGA and SEM............................... 2 - S4

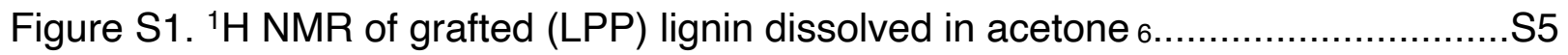

Figure S2. ${ }^{13} \mathrm{C}$ and HSQC NMR of grafted (LPP) lignin in deuterated acetone-d6.......S6

Figure S3. Full ${ }^{1} \mathrm{H} /{ }^{13} \mathrm{C}$ HSQC $2 \mathrm{D}$ NMR spectra for lignin and prepolymer products in

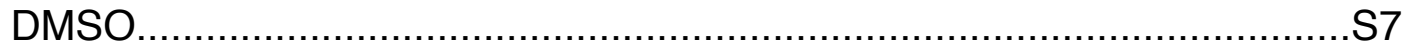

Figure S4. Aromatic and aliphatic regions from HSQC 2D NMR of lignin reagents,

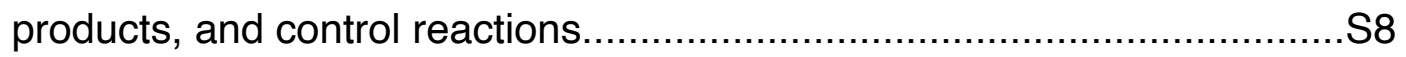

Table S1: Prominent ${ }^{1} \mathrm{H}$ NMR peaks for lignins and products dissolved in DMSO. S9

Table S2. Prominent ${ }^{13} \mathrm{C}$ NMR peaks for lignins and products dissolved in DMSO. S10

Figure S5. Example mechanism for the grafting of aromatics onto lignin S11

Figure S6. Phenol-, Lignin-, and 4-Hydroxybenzaldehyde-Formaldehyde Resins via the Electrophilic Aromatic Ortho-Substitution of $p$-Substituted Aromatics.

Table S3. Molecular weight distributions and polydispersities for lignin, prepolymer products, LNR resin, and control reaction products and solutions..........S13

Figure S7. ${ }^{H} \mathrm{H}$ NMR Spectrum of lignin resin (LNR) in DMSO.................................... 14

Figure S8. GC-FID chromatograms of lignin reaction solution before and after grafting. 


\section{Analytical Methods}

\section{Gas Chromatography}

Samples were analyzed by GC-FID by diluting $300 \mu \mathrm{L}$ of solution filtered through a 0.42 micron filter with $1 \mathrm{~mL}$ of methanol. Purchased vanillin and 4-hydroxybenzaldehyde were used as standards for analysis. The GC method used an HP-INNOWAX column on an Agilent 7890A GC and uses a carrier gas $(\mathrm{He})$ flow of $1 \mathrm{std} . \mathrm{cm}^{3} / \mathrm{min}$, an inlet temperature of $250^{\circ} \mathrm{C}$, and an injection volume of $1 \mu \mathrm{L}$. The oven temperature was initially held at 40 ${ }^{\circ} \mathrm{C}$ for $5 \mathrm{~min}$, then ramped at $10^{\circ} \mathrm{C}$ per minute to $220^{\circ} \mathrm{C}$ and held at this temperature for a further 20 min. Quantification was performed using GC-FID and calibration curves were prepared from known samples (from $0.01-5.0$ wt. \% solutions) employing previously reported methods. ${ }^{7}$ Gas chromatograms have the baseline subtracted and area collected (at $\sim 24.4$ and $\sim 31.0$ minutes for aldehydes: vanillin and 4HB respectively) in Plot2, before comparing signal to a calibration curve.

\section{Gel Permeation Chromatography}

For GPC analysis samples are dried prepared with dimethylformamide $(1.0 \mathrm{~mL})$. For prepolymer and resinous samples, the solutions are filtered through cotton to remove any precipitates. GPC was performed on an Agilent 1260 Infiniti GPC system fitted with an Agilent refractive index detector. Two columns, a $300 \mathrm{~mm}$ Polargel-M followed by a 300 $\mathrm{mm}$ Polargel-L, were used in series at $40{ }^{\circ} \mathrm{C}$. The samples were eluted with dimethylformamide at a flow rate of $0.5 \mathrm{~mL} / \mathrm{min}$. Gel permeation chromatograms are 
calibrated against a polymethylmethacrylate standard before analysis in Plot2 for peak locations.

\section{${ }^{1}{ }^{H-13} \mathrm{C} H S Q C$ Nuclear Magnetic Resonance Spectroscopy}

NMR spectra were recorded using a Bruker AVIII $500 \mathrm{MHz}$ spectrometer with a multinuclear BFFO cryoprobe. Approximately $75 \mathrm{mg}$ of the sample was dissolved in 0.75 $\mathrm{mL}$ DMSO- $\mathrm{d}_{6}$ or acetone- $\mathrm{d}_{6}$ and any remaining solids are filtered out. For the ${ }^{13} \mathrm{C}$ NMR analysis, a power-gated decoupling sequence was used to screen out the Nuclear Overhauser Effect (NOE) with the following parameters: 150 ms relaxation delay, $64 \mathrm{~K}$ data points, and 1024 scans. 2D ${ }^{1} \mathrm{H}-{ }^{13} \mathrm{C}$ HSQC NMR spectra were obtained using the hsqcedetgpsisp2.2 HSQC program. The solvent peak was used as an internal chemical shift reference point. The spectral widths were 20 ppm and 234 ppm for ${ }^{1} \mathrm{H}$ and ${ }^{13} \mathrm{C}$ dimensions, respectively, along with the following parameters: 2 second relaxation delay, 8 scans, and 256 time increments were recorded in the ${ }^{13} \mathrm{C}$ dimension. Spectra (.fid/.ser files) were analyzed in MestreNova.

\section{Thermogravimetric Analysis}

Samples were dried and weighed using a TA Instruments SDT-Q600 analyzer. Using flowing air and a ramp rate of $10^{\circ} \mathrm{C} / \mathrm{min}$ from 35 to $1000^{\circ} \mathrm{C}$, the change in mass was recorded. During analysis, the first derivative of the change in mass with respect to temperature was plotted. Raw data were converted from absolute mass to change in mass percent to more easily compare samples. 


\section{Scanning Electron Microscopy}

Grass lignin, ozonized lignin (OZL) and the LPP prepolymer product were dissolved in acetic acid (1 wt. \%) and directly placed on the head of an SEM pin. Samples are dried before being sputter coated with $5 \mathrm{~nm}$ of gold before being imaged with a FEI Versa 3D Dual Beam machine, at a voltage of $5 \mathrm{kV}$ and a spot size of $4.5 \mathrm{~nm}$. 


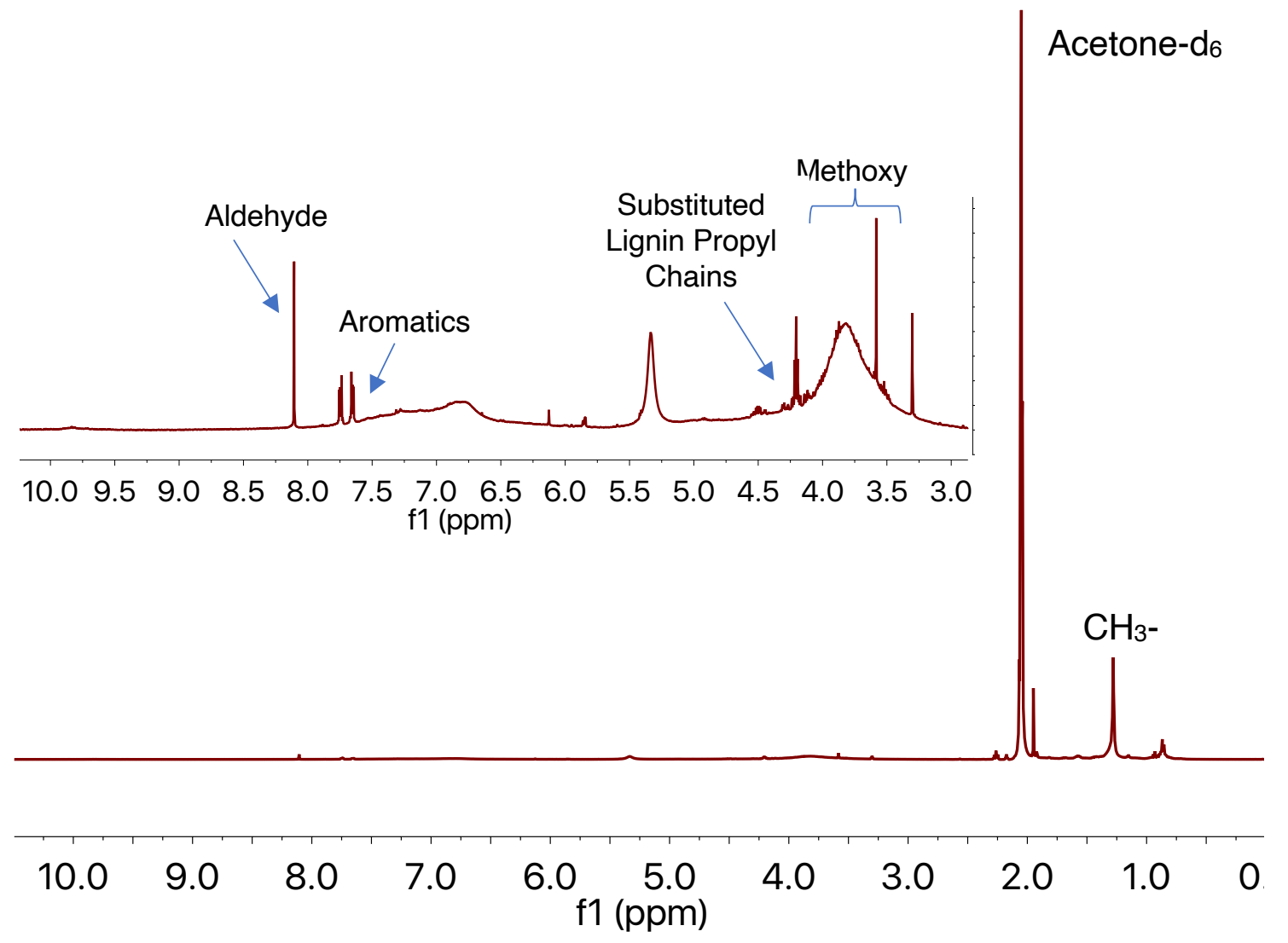

Figure S1. ${ }^{1} \mathrm{H}$ NMR of LLE grafted (LPP) lignin dissolved in deuterated acetone. Inset: Closeup of region highlighting substituted side chain and aromatics. 

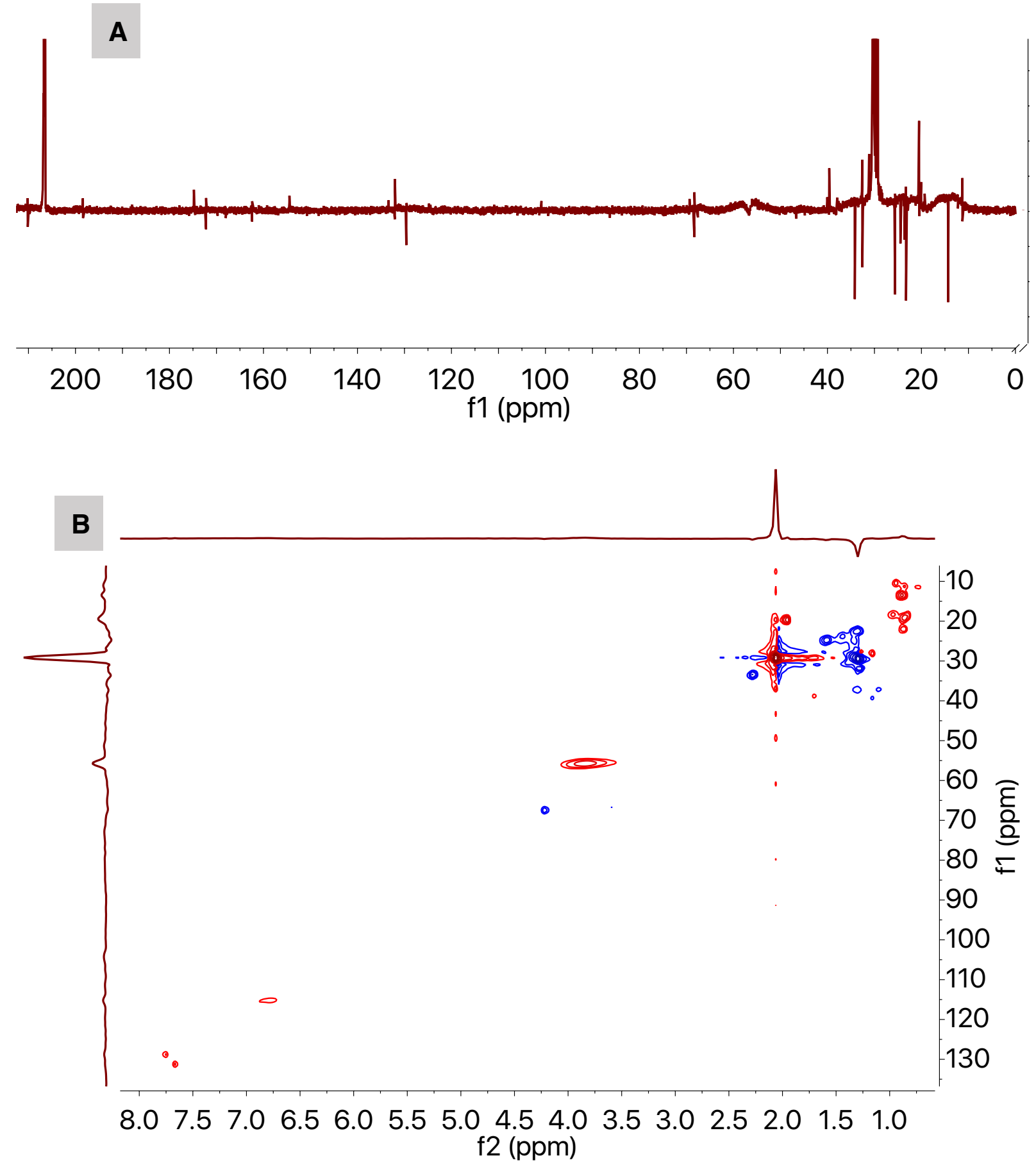

Figure S2. (A) ${ }^{13} \mathrm{C}$ DEPT NMR of grafted (LPP) lignin in deuterated acetone; $(\mathrm{B})^{1} \mathrm{H}(\mathrm{f} 2)$ ${ }^{13} \mathrm{C}$ (f1) HSQC NMR of grafted (LPP) lignin in deuterated acetone product highlighting carbon-hydrogen bonded atoms. 


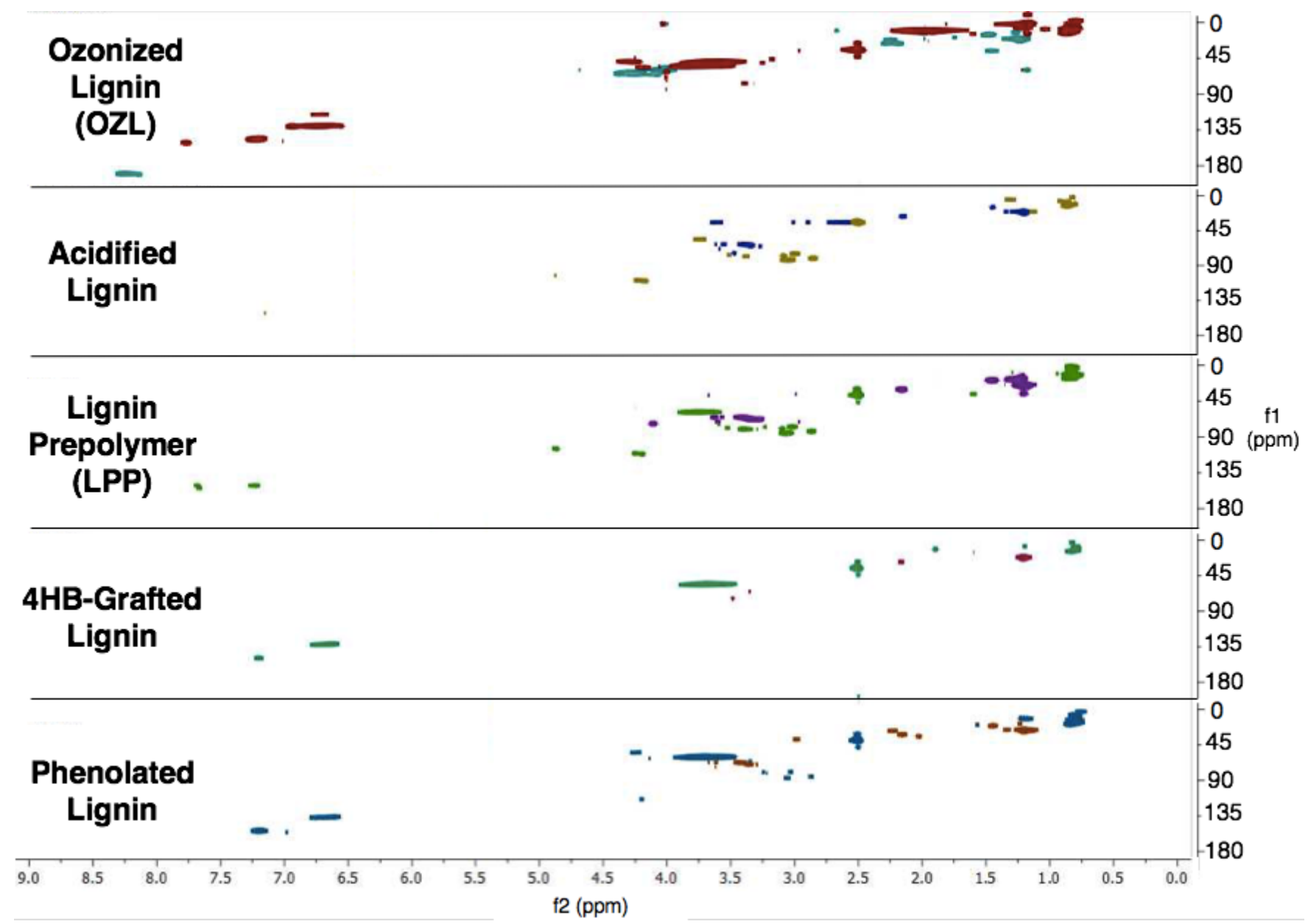

Figure S3. ${ }^{1} \mathrm{H} /{ }^{13} \mathrm{C}$ HSQC $2 \mathrm{D}$ NMR spectra for lignin, control, and prepolymer products in DMSO. 


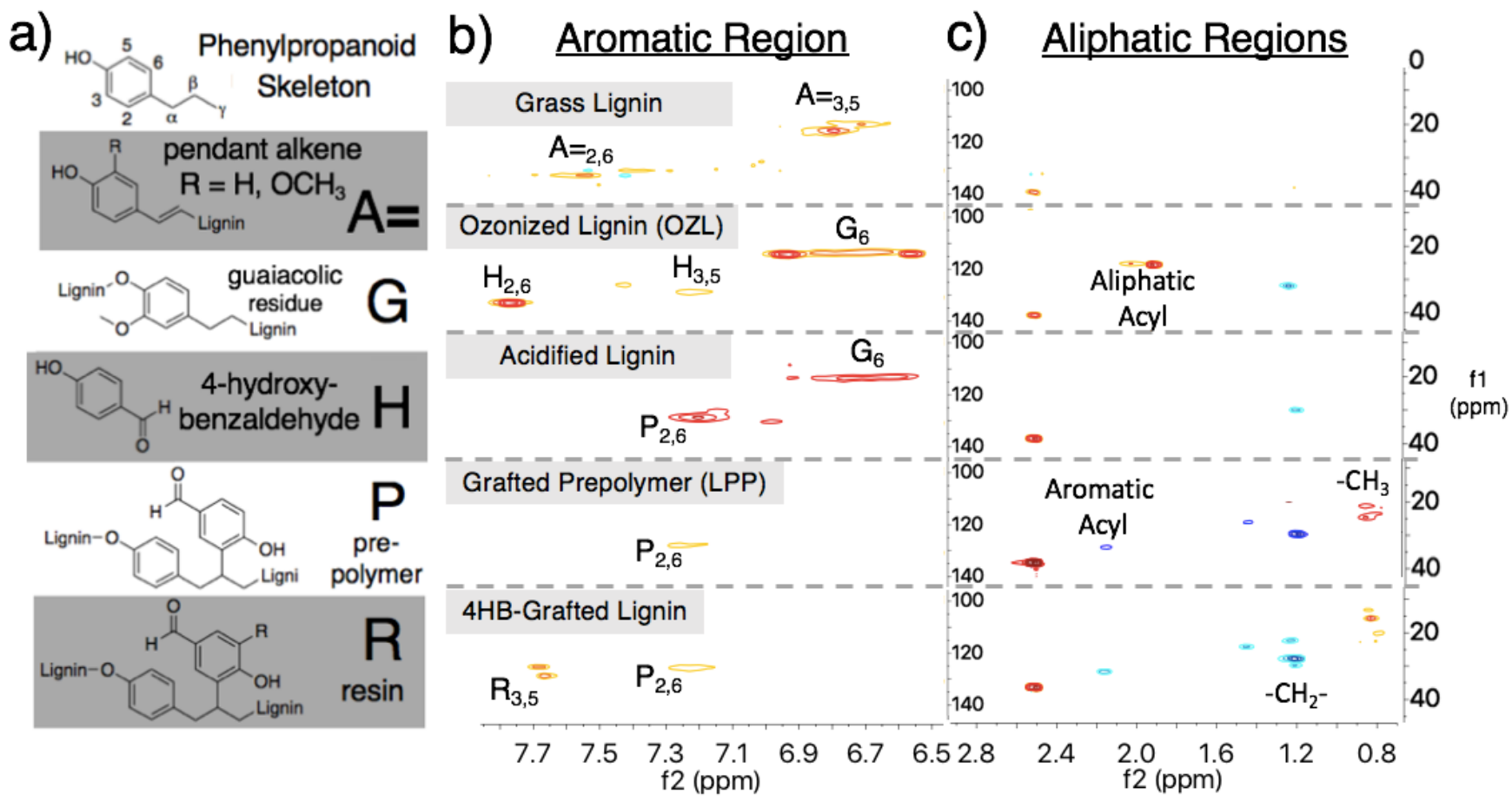

Figure S4. Closeup aromatic and aliohatic regions from 2D HSQC NMR in Figure S3 (a) Identities and structures associated with lignin, OZL, acidified grass lignin, acidified OZL or LPP, and 4HB-grafted lignin products following various treatments, along with their (b) aromatic and (c) aliphatic regions taken from ${ }^{1} \mathrm{H} /{ }^{13} \mathrm{C}$ HSQC 2D NMR in DMSO (full spectra given in SI Figure S3). 
Table S1. Prominent ${ }^{1} \mathrm{H}$ NMR peaks for lignins and products dissolved in DMSO. Peaks are denoted by their shifts $(\mathrm{ppm})$ and singlets unless otherwise denoted. Splitting: $d=$ doublet, $\mathrm{t}=$ triplet, $\mathrm{m}=$ multiplet. Other abbreviations are as noted in Figure S4. $\mathrm{V}$ denotes vanillin.

\begin{tabular}{|c|c|c|c|c|c|c|}
\hline \multicolumn{7}{|c|}{ Major ${ }^{1} \mathrm{H}$ NMR Peaks and Assignments (DMSO- $\mathrm{d}_{6}$ solvent) } \\
\hline Grass Lignin & $\begin{array}{l}\text { Ozonized } \\
\text { Lignin } \\
\text { (OZL) }\end{array}$ & $\begin{array}{l}\text { Acidified } \\
\text { Lignin }\end{array}$ & $\begin{array}{l}\text { Grafted } \\
\text { Lignin } \\
\text { (LPP) }\end{array}$ & $\begin{array}{l}\text { 4HB- } \\
\text { Grafted } \\
\text { Lignin }\end{array}$ & $\begin{array}{c}\text { Phenola } \\
\text { ted } \\
\text { Lignin }\end{array}$ & Classification \\
\hline 0.86 & 0.86 & 0.84 & 0.81 & 0.83 & 0.81 & Alkane $-\mathrm{CH}_{3}$ \\
\hline 1.24 & 1.24 & 1.21 & 1.21 & 1.20 & 1.21 & Alkane $-\mathrm{CH}_{2}$ \\
\hline 1.92 & 1.92 & & & 1.90 & 1.89 & Acetic Acid \\
\hline \multirow[t]{2}{*}{2.03} & 2.09 & 2.03 & & 2.17 & & Aliphatic Acetyl \\
\hline & 2.25 & 2.25 & & 2.53 & & Aromatic Acetyl- \\
\hline \multirow[t]{3}{*}{2.50} & 2.50 & 2.50 & 2.50 & 2.50 & 2.50 & DMSO Solvent \\
\hline & 3.18 & 3.03 & 3.16 & & 3.15 & possible resinol- $\beta$ \\
\hline & & & 3.35 & 3.37 & 3.35 & $\begin{array}{l}\beta \text {-phenyl } \\
\text { propanoid }\end{array}$ \\
\hline $\begin{array}{l}3.5-4.0 \\
3.74 \max \end{array}$ & $\begin{array}{l}3.5-3.9 \\
3.74 \max \end{array}$ & $\begin{array}{c}3.2-3.9 \\
\text { broad }\end{array}$ & $\begin{array}{l}2.9-4.0 \\
3.69 \max \end{array}$ & $\begin{array}{c}2.9-3.96 \\
\text { broad }\end{array}$ & $\begin{array}{l}3.2-4.0 \\
3.69 \max \end{array}$ & \multirow{2}{*}{$\begin{array}{c}\text { Aromatic methoxy } \\
+ \text { phenyl- } \\
\text { propanoid }\end{array}$} \\
\hline \multirow[t]{5}{*}{$3.83,3.90$} & $3.78,3.85$ & $\begin{array}{l}3.48 \\
3.77 \\
3.89 \\
\end{array}$ & $\begin{array}{c}3.48,3.60 \\
3.89\end{array}$ & $\begin{array}{c}3.48,3.78 \\
3.89\end{array}$ & $\begin{array}{l}3.49 \\
3.90\end{array}$ & \\
\hline & & & & & $4.11 \mathrm{t}$ & phenyl propanoid \\
\hline & & $\begin{array}{l}4.19 \mathrm{~d} \\
4.24 \mathrm{~d} \\
\end{array}$ & $4.17 \mathrm{~m}$ & $\begin{array}{l}4.20 \\
4.25 \\
\end{array}$ & $\begin{array}{l}4.19 \mathrm{~d} \\
4.25 \mathrm{~d} \\
\end{array}$ & $\begin{array}{c}\text { Y-phenyl } \\
\text { propanoid }\end{array}$ \\
\hline & & & 4.85 & 4.85 & 4.85 & $\begin{array}{c}\text { Hydroxy-phenyl } \\
\text { propanoid - } \mathrm{CH}^{\star}- \\
\mathrm{OH}_{-}\end{array}$ \\
\hline & 6.65 & 6.61 & $\begin{array}{c}6.62 \\
\text { broad }\end{array}$ & & $\begin{array}{c}6.62 \\
\text { broad }\end{array}$ & $\begin{array}{l}\text { Alkene } \\
=\mathrm{C}-\mathrm{H}\end{array}$ \\
\hline $\begin{array}{l}6.79 \mathrm{~A}=\beta \\
7.52 \mathrm{~A}=\alpha\end{array}$ & $\begin{array}{c}6.93 \mathrm{~d} \\
\mathrm{H}_{3,5} \\
7.01 \mathrm{~V}_{5} \\
7.43 \mathrm{~V}_{2,6} \\
7.77 \mathrm{H}_{2,6} \\
\end{array}$ & $7.20 \mathrm{P}$ & $7.15 \mathrm{P}_{2,6}$ & $\begin{array}{l}7.21 \mathrm{P} \\
7.69 \mathrm{R}\end{array}$ & $7.20 \mathrm{P}$ & $\begin{array}{l}\text { Aromatic } \\
\text { Hydrogens }\end{array}$ \\
\hline 8.15 & 8.14 & & 8.21 & & & Phenolic -OH \\
\hline
\end{tabular}


Table S2. Prominent ${ }^{13} \mathrm{C}$ NMR peaks in lignin and lignin products dissloved in DMSO. Peaks are denoted by their shifts (ppm). V stands for vanillin, and Lignin-H denotes a lignin-bonded $\mathrm{H}$-unit. All other abbreviations are as noted in Figure S4.

\begin{tabular}{|c|c|c|c|c|c|}
\hline \multicolumn{6}{|c|}{ Major ${ }^{13} \mathrm{C}$ NMR Peaks and Assignments (DMSO-d6 solvent) } \\
\hline $\begin{array}{c}\text { Ozonized } \\
\text { Lignin (OZL) }\end{array}$ & $\begin{array}{l}\text { Acidified } \\
\text { Lignin }\end{array}$ & $\begin{array}{c}\text { Grafted } \\
\text { Lignin } \\
\text { (LPP) }\end{array}$ & $\begin{array}{c}\text { 4HB } \\
\text { Grafted } \\
\text { Lignin } \\
(4 \mathrm{HB})\end{array}$ & $\begin{array}{l}\text { Phenolated } \\
\text { Lignin }\end{array}$ & Classification \\
\hline 20.55 & 21.25 & 21.76 & 21.25 & & Acetyl- \\
\hline 39.52 & 39.52 & 39.52 & 39.52 & 39.52 & DMSO Solvent \\
\hline 48.65 & & & & & possible resinol- $\beta$ \\
\hline 59.55 & & & 51.11 & & phenyl-propanoid \\
\hline 55.68 & 55.68 & 56.19 & 55.68 & 55.68 & Aromatic Methoxy \\
\hline & $\begin{array}{c}62.71 \\
\mathrm{G} \gamma\end{array}$ & & $\begin{array}{c}62.71 \\
G \gamma\end{array}$ & $\begin{array}{c}62.71 \\
\mathrm{G} \gamma\end{array}$ & \multirow{2}{*}{$\begin{array}{c}\alpha, \beta, \gamma-\text { phenyl- } \\
\text { propanoid }\end{array}$} \\
\hline & $\begin{array}{c}82.73 \\
G_{\beta}\end{array}$ & & $\begin{array}{c}71.84 \\
\mathrm{G}_{\alpha}\end{array}$ & & \\
\hline $\begin{array}{c}114.71 \\
\mathrm{G}_{5}\end{array}$ & & & & & \multirow{6}{*}{ Aromatic Carbons } \\
\hline $\begin{array}{c}115.06 \\
V_{5}\end{array}$ & $\begin{array}{c}115.06 \\
G_{5}\end{array}$ & & & $\begin{array}{c}115.76 \\
\mathrm{G}_{5}\end{array}$ & \\
\hline $\begin{array}{c}115.76 \\
H_{3,5}\end{array}$ & & & $\begin{array}{c}128.41 \\
\mathrm{H}_{2,6}\end{array}$ & $\begin{array}{c}\delta 128.37 \\
\mathrm{H}_{2,6}\end{array}$ & \\
\hline $\begin{array}{c}128.41 \\
\mathrm{H}_{2,6}\end{array}$ & $\begin{array}{c}128.76 \\
\mathrm{H}_{2,6}\end{array}$ & & $\begin{array}{c}128.76 \\
\mathrm{H}_{2,6}\end{array}$ & & \\
\hline $\begin{array}{c}129.81 \\
A=2,6\end{array}$ & & $\begin{array}{c}129.27 \\
\mathrm{R}\end{array}$ & $\begin{array}{c}129.11 \\
\mathrm{R}\end{array}$ & & \\
\hline $\begin{array}{c}131.92 \\
\text { Lignin- } \mathrm{H}_{2,6}\end{array}$ & & $\begin{array}{l}145.08 \\
C_{5} \text { in 5- } \\
5 \text { ' units }\end{array}$ & $\begin{array}{l}131.57 \\
C_{1} \text { in } 5- \\
5^{\prime} \text { ' units }\end{array}$ & & \\
\hline
\end{tabular}



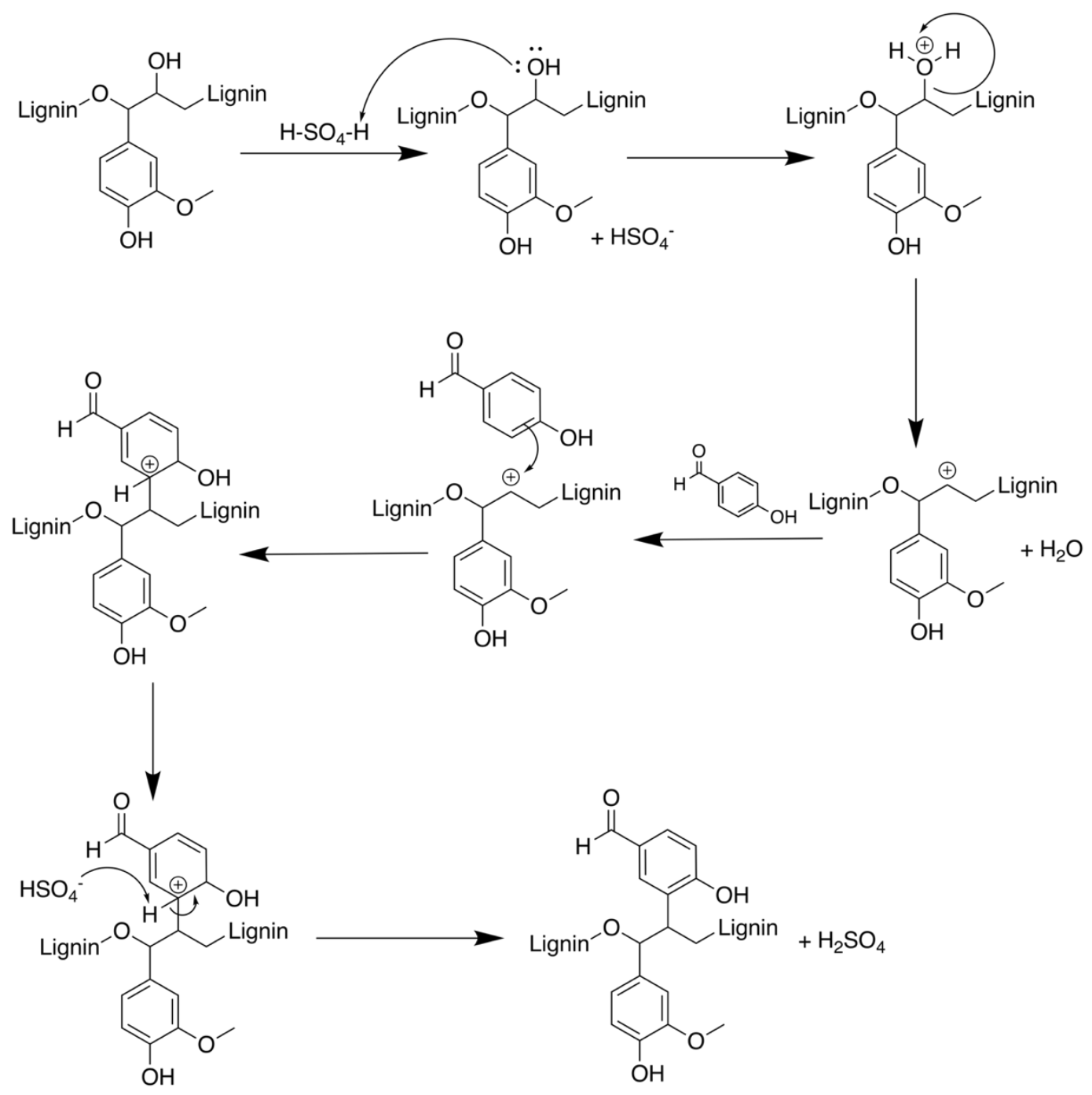

Figure S5: Example mechanism for the grafting of aromatics onto grass lignin: The electrophilic aromatic Substitution of 4 -Hydroxybenzaldehyde and the $\beta$-position on propyl side chain. Note that a bond to 'Lignin' represents possible linkages to molecular and macromolecular phenylpropanoids. 


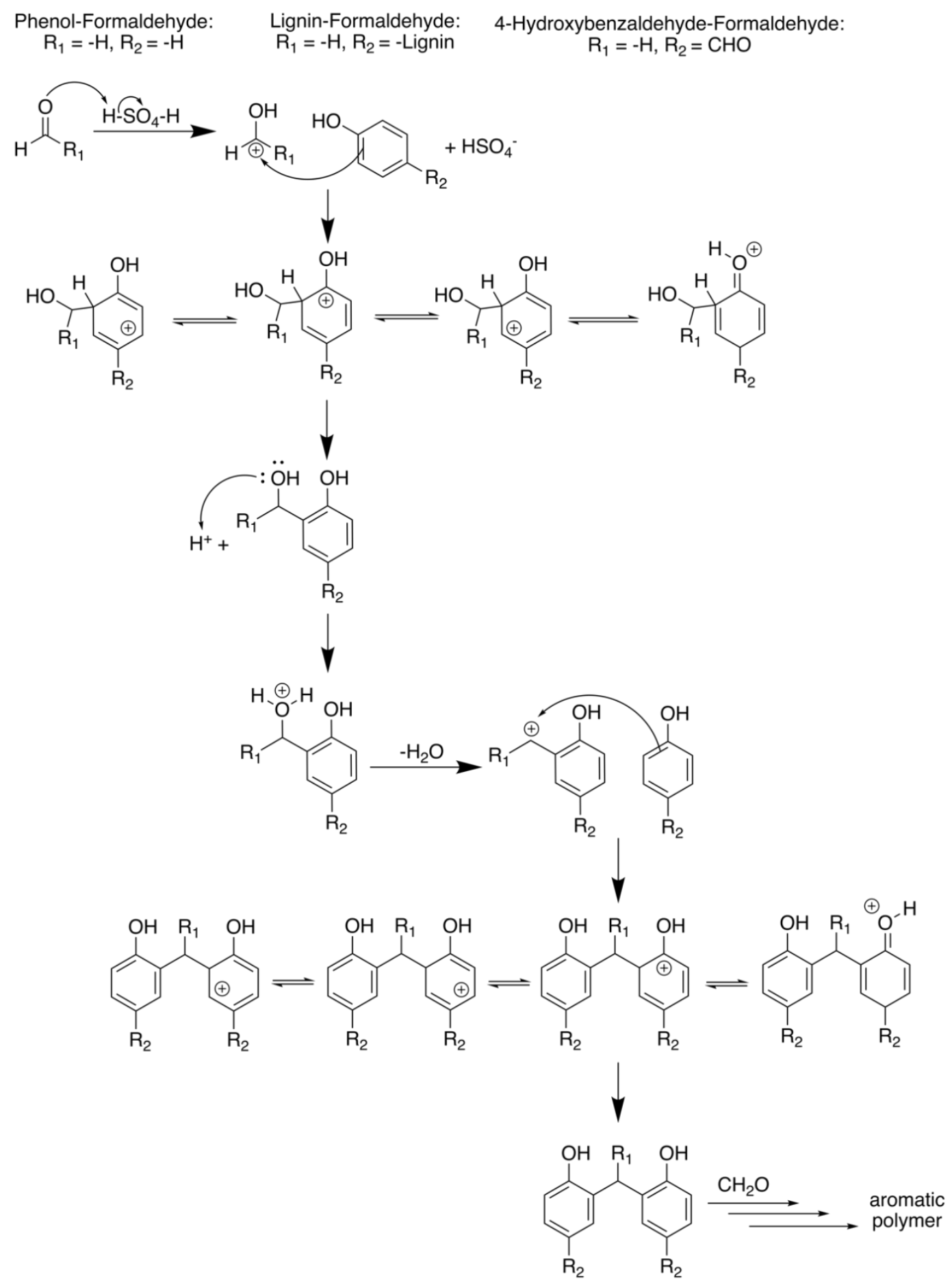

Figure S6. Phenol-, Lignin-, and 4-Hydroxybenzaldehyde-Formaldehyde Resins via the Electrophilic Aromatic Ortho-Substitution of $p$-Substituted Aromatics. Note: Phenol will preferentially undergo a para-substitution. 
Table S3. Molecular weight distributions and polydispersities of lignin and lignin-derived products corresponding to Figure 3 . Note that values are not reported for the LPP supernantant as the solute concentration is below the limit of detection of the instrument.

\begin{tabular}{|c|c|c|c|c|}
\hline Lignins and Products & $\begin{array}{l}\text { Label in } \\
\text { Figure } 3\end{array}$ & $\begin{array}{c}\text { Number } \\
\text { Average } \\
\text { Molecular } \\
\text { Weight (Mn) }\end{array}$ & $\begin{array}{c}\text { Weight } \\
\text { Average } \\
\text { Molecular } \\
\text { Weight (Mw) }\end{array}$ & $\begin{array}{l}\text { Poly- } \\
\text { dispersity } \\
\text { Index } \\
\text { (PDI) }\end{array}$ \\
\hline Ozonized Lignin (OZL) & $\mathrm{A} 1$ & 29,800 & 69,000 & 2.31 \\
\hline LMW Lignin Extract from OZL (LLE) & $\mathrm{A} 2$ & 8,270 & 22,200 & 2.68 \\
\hline HMW Lignin Solids from OZL (HLS) & A3 & 49,900 & 93,700 & 1.88 \\
\hline Grafted Prepolymer (LPP) & A4 & 98,400 & 181,000 & 1.84 \\
\hline Lignin 'Novolac' Resin (LNR) & A5 & 182,000 & 640,000 & 3.52 \\
\hline Grass Lignin & B1 & 29,500 & 72,700 & 2.46 \\
\hline Acidified Grass Lignin & B2 & 58,900 & 126,000 & 2.13 \\
\hline Acidified HLS & B3 & 91,200 & 287,000 & 4.53 \\
\hline 4HB-Grafted OZL & B4 & 80,000 & 191,000 & 2.38 \\
\hline Phenolated OZL & B5 & 84,400 & 501,000 & 5.94 \\
\hline LPP Reaction Supernatant & $\mathrm{C} 1$ & - & - & - \\
\hline $\begin{array}{l}\text { Unreacted Phenol-Formaldehyde } \\
\text { Mixture }\end{array}$ & $\mathrm{C} 2$ & 110 & 170 & 1.55 \\
\hline $\begin{array}{c}\text { Phenol-Formaldehyde } \\
\text { Polymerization }\end{array}$ & C3 & 2,580 & 15,900 & 6.17 \\
\hline 4HB-Formaldehyde Polymerization & $\mathrm{C} 4$ & 550 & 3,580 & 6.45 \\
\hline
\end{tabular}

$\frac{\frac{\sum_{i} N_{i} M W_{i}}{N_{i}}}{\text { Number Average Molecular Weight }\left(M_{N}\right)=\frac{\sum_{i} N_{i}\left(M W_{i}\right)^{2}}{\sum_{i} N_{i} M W_{i}}}$




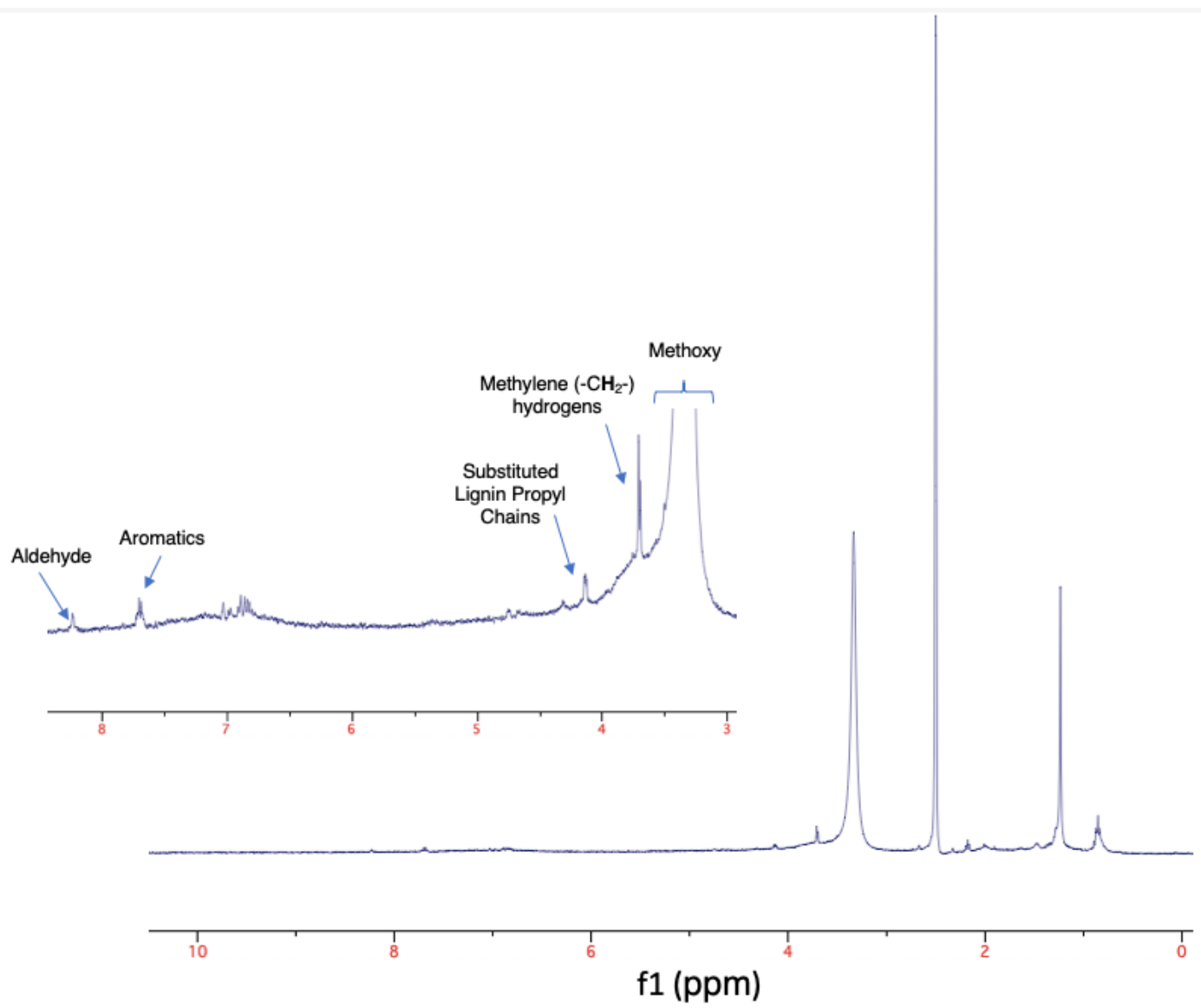

Figure S7. ${ }^{1} \mathrm{H}$ NMR spectrum of LNR from the reaction of LPP and formaldeyde. The peaks appearing at $\sim 3.7 \mathrm{ppm}$ are indicative of hydrogens in the methylene bridge between aromatic rings. 

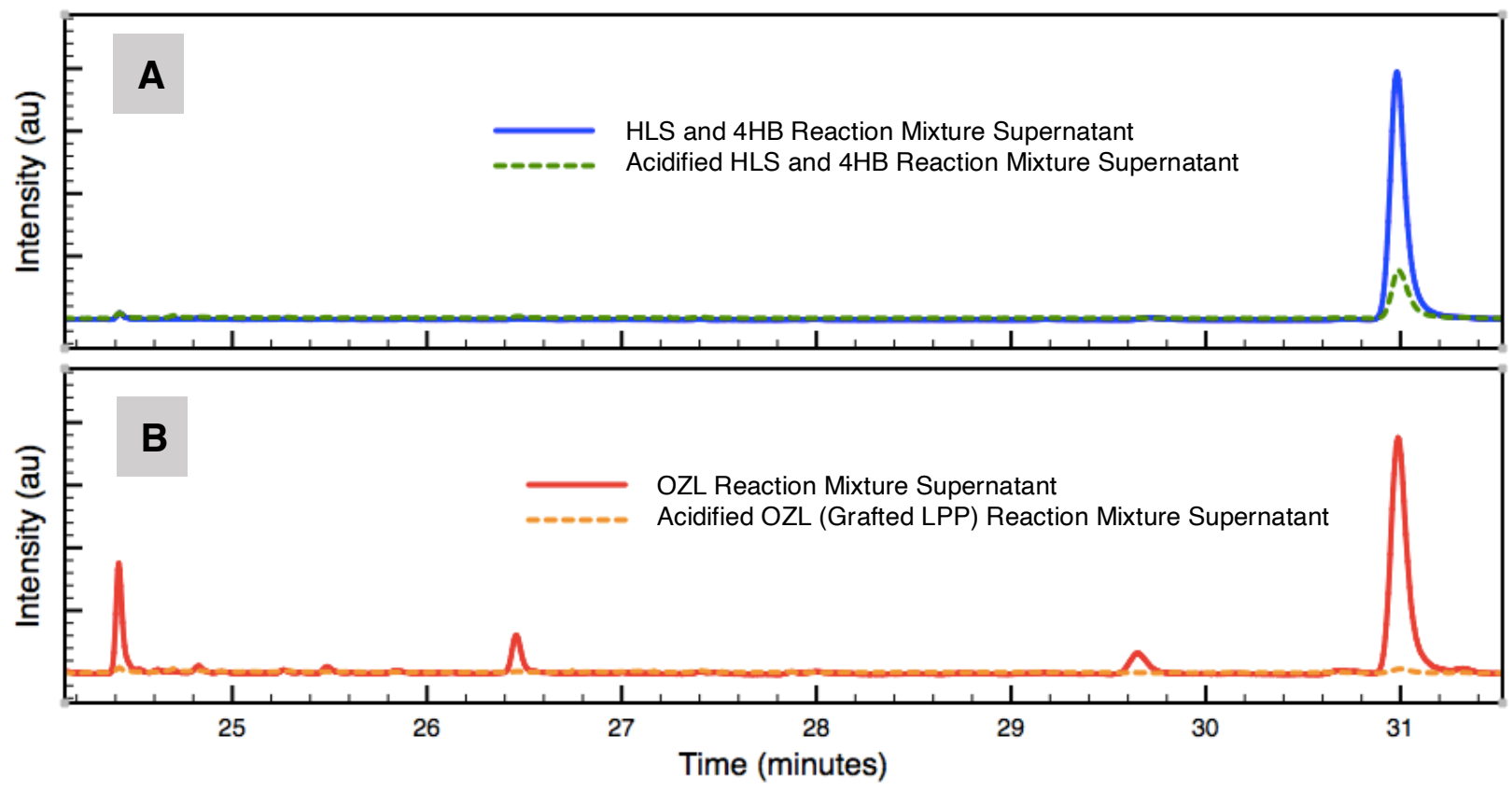

Figure S8. Gas Chromatograph/FID chromatogram of reaction mixture supernatant before and after acidification of (A) HLS and purchased 4HB; and (B) ozonized lignin mixture (OZL) comprised of both HLS and LLE fractions. Peaks at 24.4, 26.4, 31 represent vanillin, hydroxycinnamic acid, and $4 \mathrm{HB}$ as determined with external standards. 